\title{
Circuit
}

Musiques contemporaines

\section{Ruer dur et faire entendre}

Dix fragments sur l'art politique de Zappa

Kick Hard and Make Them Hear

Ten snatches on Zappa's political art

\section{Michel F. Côté}

Volume 14, numéro 3, 2004

Frank Zappa : 10 ans après

URI : https://id.erudit.org/iderudit/902329ar

DOI : https://doi.org/10.7202/902329ar

Aller au sommaire du numéro

Éditeur(s)

Les Presses de l'Université de Montréal

ISSN

1183-1693 (imprimé)

1488-9692 (numérique)

Découvrir la revue

Citer cet article

Côté, M. F. (2004). Ruer dur et faire entendre : dix fragments sur l'art politique de Zappa. Circuit, 14(3), 91-102. https://doi.org/10.7202/902329ar
Résumé de l'article

Frank Zappa fut un compositeur engagé. Inlassable commentateur de l'actualité sociale et politique, c'est avec audace qu'il a toujours créé ses charges musicales et verbales. Fils légitime de dada, Zappa a cultivé la liberté d'expression avec ferveur. Dès ses débuts en 1966, la prise de parole est directe et intense, sans ménagement.

Composé de dix courts chapitres (de F à A), ce texte propose un survol en polaroid de l'art politique de ce guitariste guérillero et rieur. 


\title{
Ruer dur et faire entendre (Dix fragments sur l'art politique de Zappa)
}

\author{
Michel F. Côté
}

"I spent the first five or six years of my life with cotton hanging out of my ears -

yellow, from olive oil. "'

Frank Zappa

\section{Vestibule de l'oreille interne}

Le guitariste n'était pas sensationnel, le chanteur était moyen et le compositeur, habile. Il le savait mieux que nous. Son doute fut prolifique : l'artiste fut un inventeur d'exception; magnifiquement plus accompli qu'uniquement musicien. Pendant trente ans, avec humour, il a pris son job très au sérieux. Opiniâtre et intraitable, il fut monstrueusement pertinent.

II sera très peu question de musique ici. D'autres s'en occuperont.

Ce texte porte sur Frank Zappa, personnage protéiforme et incandescent, artiste américain qui préfigura de quelques années l'agitation qui bousculera bientôt l'Occident bienheureux. Sur la pochette de "We're Only in It for the Money", à propos de ce disque, il écrit: "THIS WHOLE MONSTRUOSITY WAS CONCEFVED \& EXECUTED BY FRANK ZAPPA AS A RESULT OF SOME UNPLEASANT PREMONITIONS, AUGUST THROUGH OCTOBER 1967²."

AMERICAN WAY
How did it start?
Thousands of creeps
Killed in the park
AMERICAN WAY
Try and explain
Scab of a nation
Driven insane

(Concentration Moon, "We're Only in It for the Money", 1968³.)
1. The Real Frank Zappa Book, Frank Zappa avec Peter Occhiogrosso, New York, Poseidon Press/Simon \& Schuster, Inc., 1989, p. 19. Il est idiot d'avoir à présenter une traduction française des citations plutôt que le texte anglais d'origine; nous y perdons en musicalité. J'ai donc préféré, pour le style verbal unique de Zappa, le chant de sa langue, sa rythmique remarquablement efficace et la précision du sens, offrir les mots d'origine plưôt qu'une traduction nécessairement imparfaite. La traduction française de The Real Frank Zappa Book, très française, réalisée par Jean-Marie Millet I Zappa par Zappa, Frank Zappa avec la collaboration de Peter Occhiogrosso, L'Archipel, Paris, 2000), a servi de base à quelques-unes des traductions que vous lirez dans les notes. J'ai apporté quelques corrections ici et là.

Traduction : «J'ai passé les cinq ou six premières années de ma vie avec, dans les oreilles, de petits bouts de coton jaunis à I'huile d'olive."

2. Traduction: «Cette monstruosité a été entièrement conçue et dirigée par Frank Zappa d'août à octobre 1967; elle est le résultat de prémonitions désagréables. * 
Zappa est un moderne. J'aime bien penser à Satie quand je pense à Zappa, et vice versa. Pour l'inventivité, l'ironie, l'engagement, la transgression, dada et une audace étonnante.

"Rien à voir entre ce Français lunatique et notre Américain énergique», diront certains. Basta!, Cage serait de mon avis.
3. Traduction : "AMERICAN WAY/ Comment cela a-til débuté? / Des milliers d'êtres infects/Tués dans le parc/AMERICAN WAY/Essayer d'expliquer/Gangrène d'une nation/Poussée à la démence. »

\section{Plutôt tôt que tard}

Agitateur né en 1940, Frank Zappa est rapidement devenu un porte-parole turbulent de l'autocritique sociale américaine post-fiffies. Pur produit de cette effervescence étasunienne d'après-guerre, celle de la droite puritaine et du protestantisme prospère made in USA, il fut en constante réaction devant l'hypocrisie conquérante de ce nouvel empire politique et culturel. Sa personnalité flamboyante, son goût pour dada, ses prises de positions légèrement anarchistes, sa constante guérilla critique contre cette Amérique des lieux communs et de la débilité ambiante, puis cette façon unique de se créer des ennemis là où il était pourtant accueilli en héros, feront de lui, dès 1965, à l'âge de 25 ans, un créateur sans complaisance, un personnage désormais incontournable.

Pendant que le mouvement hippie embrasait la côte Ouest et que les chevelures s'allongeaient au fil des heures, Zappa, imperturbable et cynique, s'amusait du peu de vraisemblance de cette révolution d'amateur. II n'y a qu'à relire les textes des chansons de "Freak Out! » (1966), "Absolutely Free" (1967) ou "We're Only in It for the Money» (1968) pour constater l'acharnement avec lequel il s'attaqua très tôt aux illusions néoromantiques de ses contemporains poilus : la paix et l'amour n'étaient que de beaux idéaux simplistes, encore fallait-il être capable d'articuler un discours susceptible de rendre l'absolu nécessité de corriger la trajectoire d'une Amérique aveuglément triomphante.

Insistons : Zappa n'accepte aucune excuse au manque de convictions des aspirations révolutionnaires juvéniles de ses contemporains. Face à la faiblesse de cette insurrection psychédélique et fadasse, en réaction à l'agitation molle de ces fils à papa en overdose, Zappa forge des textes satiriques sulfureux, invente des alliages musicaux stylistiquement télescopés, et imagine un look trash à ce point déjanté qu'il donne, en contraste, une allure bon enfant au stéréotype vestimentaire hippie alors émergent.

Pragmatique convaincu, Zappa a toujours affiché une irréductible honnêteté intellectuelle; aucune complaisance chez lui : il ne peut être question d'entretenir une apparence de contre-culture tout en jouant le jeu de la normalité. II réagira de manière particulièrement violente aux diktats des magnats de l'industrie du disque et du spectacle qui bavent soudainement d'appétence devant les perspectives 
réjouissantes qu'offre l'explosion de ce nouveau marché pour teenagers en mal d'identification culturelle.

La détermination de son engagement politique est singulière dans I'histoire de la musique américaine de la seconde moitié du Xx siècle. Ses nombreux textes polémiques en font foi (Say Cheese... publié à l'intérieur de la jaquette du disque "You Are What You ls" [1981] est un excellent exemple de la production littéraire de Zappa; article à l'origine écrit pour Newsweek, mais refusé par ce magazine, on peut y savourer l'insolence pertinente de son auteur. En voici un extrait : "Our mental health has been in a semi-wretched condition for quite some time now. One of the reasons for this distress, aside from CHOOSING CHEESE as a way of life, is the fact that we have (against some incredibly stiff competition) emerged victorious as the biggest bunch of liars on the face of the planet. No society has managed to invest more time and energy in the perpetuation of the fiction that it is moral, sane and wholesome than our current crop of Modern Americans. "4)

Zappa a farouchement défendu la liberté d'expression, la sienne : celle de l'artiste, mais aussi celle du citoyen. Peu de musiciens/compositeurs ont exigé de manière aussi constante le respect du premier amendement de la Constitution américaine ${ }^{5}$, allant jusqu'à défendre ce droit devant le Sénat et les différentes cours de justice, et cela plus souvent qu'à son tour.

Zappa était nécessaire, férocement indépendant et sans compromis. Les Américains le négligeaient, ils sont maintenant peut-être nombreux à s'ennuyer innocemment de cet artiste fermement engagé. In the Land of the Politically Correct et du mensonge vertueux, il était un des seuls à ruer dur.
4. Traduction: "Depuis un certain temps, notre santé mentale est dans un état lamentable. Une des raisons de cette détresse, outre le fait d'avoir CHOISI DE SOURIRE comme façon de vivre, repose sur le fait que nous sommes victorieusement devenus (malgré une compétition incroyablement vigoureuse) le plus grand rassemblement de menteurs existant sur cette planète. Aucune société n'est parvenue à investir autant de temps et d'énergie à permettre l'illusion qu'elle est morale, saine et sensée que notre troupeau actuel d'Américains Modernes. "

5. Extrait de l'article 1 du premier amendement : « Le Congrès s'interdira de promulguer toute loi qui tendrait à établir une religion, ou interdire l'exercice d'une religion, ou limiter la liberté de parole ou celle de la presse [...]. »

\section{Desiderata protéiformes}

Miles Davis (1991), John Cage (1992), puis Frank Zappa (1993) : trois disparitions successives qui précipitent la fin de l'histoire musicale du XXe siècle. Ici, je ne signifie pas l'histoire musicale "officielle" telle qu'elle est généralement racontée par les académiciens aux vues consanguines; je désigne plutôt celle, plus réaliste, que tout mélomane sensible à son époque peut observer. Davis, Cage et Zappa, trois musiciens américains terriblement complémentaires (personnages de soap : un black, boxeur et rageur; un blanc, gay épris de philosophie orientale; et un fils d'immigrant sicilien, guitariste de rhythm \& blues) : il n'est pas exagéré d'affirmer qu'ils forment à eux seuls un triangle pouvant contenir une très large part des investigations musicales (et politiques) influentes des cinquante dernières années. Leurs desiderata respectifs s'additionnent merveilleusement : épris de liberté, Davis entretient une passionnelle préoccupation pour l'émancipation des Noirs américains, sa fureur est en sourdine, sans implication directement politique, son cri (stratégique) n'en est pas 
moins puissant; Cage se positionne en anarchiste, il souhaite la fin de l'État, proclame la souveraineté de l'intelligence et l'inutilité du pouvoir politique; Zappa mesure le désastre, constate l'ampleur de l'effondrement social, traque les démagogues, malmène la cupidité des politiciens, accumule les mises en accusations et provoque ses interlocuteurs jusqu'en cour.

Trois compositeurs prolifiques et politiques pour une étonnante cohérence dans une apparente disparité esthétique. Une histoire des États-Unis pourrait s'écrire à l'aide de ce prisme triangulaire.

"On n'échappe pas à la dimension politique de l'œuvre. Les artistes qui croient à la possibilité de produire un travail esthétique en dehors de la sphère politique se trompent gravement. La plupart du temps, ils prétendent éviter la compromission politique pour sombrer plus facilement dans celle de la marchandisation. Car les institutions disposent d'un formidable pouvoir de nuisance en n'accueillant pas dans leurs enceintes des œuvres qui les interrogent, les critiquent ou les mettent seulement en cause $^{6}$. "Onfray résume l'absence de dilemme, comme si l'artiste disposait d'un choix : politique ou apolitique? Divergent ou convergent? II n'y a que les illusionnistes pour prétendre créer en dehors de l'ordre social. Davis, Cage et Zappa n'escamotent rien, ce sont des chercheurs passionnément ancrés dans la réalité sociale de leur époque.

$C^{\prime}$ est donc à la source de cette curieuse trilogie de compositeurs que je me suis toujours abreuvé musicalement, et autrement. Des trois, Zappa me fut longtemps le plus familier, le plus naturel; il est certainement le compositeur qui m'a offert le plus de plaisir. Ludique et lubrique, son univers à la fois débridé, prospectif et virtuose a toujours eu cette remarquable qualité d'unir plaisir et intelligence. Une seule soirée en compagnie de l'univers musical et intellectuel de Zappa me semblera toujours plus saine et vivifiante, plus contagieuse et engageante que toutes les pages rigoristes écrites sur la Neuen Musik.

Si Miles a créé un champ de force allant de Parker aux Black Panters et de Hendrix à Sartre; Cage, un autre allant de Satie à Suzuki et de Duchamps à Schœnberg; Zappa a créé un champ gravitationnel allant de Varèse aux Beach Boys et de Beefheart à Reagan...

Il n'a épargné personne.
6. ONFRAY, Michel (2003), Archéologie du présent, Manifeste pour une esthétique cynique, Paris, Grasset/Adam Biro, p. 100.

\section{Pinch bien senti}

"INTERCONTINENTAL ABSURDITIES (founded 1968) is a company dedicated to Dada in Action. In the early days, I didn't even know what to call the stuff my life was made of. You can imagine my delight when I discovered that someone in a distant land had the same idea - AND a nice, short name for it?

Dada est omniprésent chez Zappa, musicalement, verbalement et visuellement. Trois exemples parmi mille.
7. The Real Frank Zappa Book, op. cit., p. 255. Traduction : «INTERCONTINENTAL ABSURDITIES (fondé en 1968) est une compagnie qui se consacre au concept de dada en actes. Au début, je ne savais pas comment nommer le fouillis de ma vie. Imaginez mon ravissement quand j'ai découvert que quelqu'un, d'un pays lointain, avait eu la même idée - et trouvé un jolie nom, tout court, pour dire ça.» 
Dans le riche champ des archétypes sonores brillamment exploités par Zappa, le rire (souvent jaune...) semble être un morceau de choix. Dès les premières productions sur disque, le rire "manière Zappa " commente l'ordre des choses. Chez lui, l'hilarité est un leitmotiv sonore obsessionnel qui semble surgir du chaos à tout moment. Rires accélérés, hystériques, (déçus) et ingénus : I'Amérique (au travers des protagonistes récurrents de la constellation "zappaïenne"| rigole de se voir si belle, si naïvement puissante; elle s'extasie devant les perspectives de ce nouvel empire bienfaiteur et rieur. Alors rions...

Le rire de Zappa nous rappelle sans cesse qu'il n'y a rien de très drôle avec les États-Unis et leurs hantises : face à l'obscurantisme ravageur des sornettes créationnistes, mourir de rire semble être une alternative plus acceptable que de mourir de honte. "Dada est idiot. Le véritable dadaïste, il rit, il rit», affirme le manifeste dada rédigé par Richard Huelsenbeck pour la soirée conférence du 12 avril 1918, à Berlin. l'idiotie "zappaïenne" et son rire éberlué est en accord avec dada; elle en est d'ailleurs une des seules manifestations spécifiquement américaines. Tardive, mais aussi prophétique, elle préfigure cette place centrale accordée à la rigolade généralisée comme choix culturel : l'empire des humoristes québécois n'en estil pas un saisissant exemple local...

Chez Zappa, dada se fait aussi guide distingué. Ses mots vous offrent des solutions, idiotes et cyniques. Vous désirez devenir compositeur? Voici ce que Zappa vous recommande :

\section{JUST FOLLOW THESE SIMPLE INSTRUCTIONS :}

\section{[1] Declare your intention to create a "composition."}

[2] Start a piece at some time.

[3] Cause something to happen over a period of time (it doesn't matter what happens in your "time hole" - we have critics to tell us whether it's good or not, so we won't worry about that part).

[4] End the piece at some time lor keep it going, telling the audience it is a "work in progress").

[5] Get a part-time job so you can continue to do stuff like this. ${ }^{8}$

Sensible à l'image (ne s'est-il pas très tôt activement intéressé au cinéma), Zappa ne laisse visuellement rien au hasard (sa longue association avec Cal Schenkel est à ce titre éloquente). D'un strict point de vue iconographique, Zappa a fait des merveilles : qui ne reconnaît pas cette luxuriante moustache associée à un pinch bien senti ? À contre-courant, il a transgressé avec acidité le look généralement admis. La pochette de "We're Only in It for the Money" (1968) en est un excellent exemple: corrosive relecture de la célèbre pochette de "Sgt. Pepper's Lonely Hearts Club Band " (1967) - disque intouchable d'un groupe unanimement célébré, The Beatles Zappa et Schenkel y parodient minutieusement chaque détail de la pochette, recto verso. Là où les Beatles sont gentils, Zappa devient vindicatif; là où les Beatles sont
8. The Real Frank Zappa Book, op. cit., p. 162.

Traduction : « INSTRUCTIONS SIMPLES À SUIVRE : [1] Annoncez votre intention de créer une "composition". [2] Commencez la composition à un moment donné. [3] Faites en sorte que quelques chose se produise pendant un laps de temps (peu importe ce qui se produit pendant le "trou temporel" qui est vôtre - les critiques sont là pour vous signaler ce qui est bon et ce qui ne l'est pas, donc aucun souci à se faire de ce côtél. [4] Terminez la composition à un autre moment donné lou continuez, mais prévenez le public que la composition est encore en progression). [5] Prenez un emploi à mi-temps de façon à pouvoir poursuivre ce type d'activité. » 
habillés de costumes colorés parodiant l'uniforme des fanfares militaires de la fin du XIX siècle, les Mothers se travestissent hideusement en vieilles dames indignes. Le titre du disque à lui seul est un affront, générant par cette charge sémantique et visuelle la fureur de Paul McCartney (seul John Lennon aura l'humour nécessaire pour célébrer cette satirel.

Aucune complaisance chez Zappa et un maximum d'irévérence inégalée : "We didn't have long hair, we didn't have band uniforms and we were ugly as fuck. We were in the Biblical sense of the word, UNEMPLOYABLE. ."
9. Ibid., p. 66. Traduction : «Nous n'avions ni cheveux longs, ni uniforme, et nous étions irrémédiablement laids. Nous étions, au sens biblique du terme, INEMPLOYABLES. »

\section{Two Hundred Years Old}

Commentateur inlassable, il a écrit beaucoup de chansons, plus de trois cents (décompte approximatif des chansons enregistrées sur disque). C'est une énorme production dans laquelle Zappa se fait l'analyste de la société américaine, de ses phobies et de sa détresse :

Si, comme les dadaïstes, Zappa ne respecte rien, c'est que comme eux, il considère que rien n'est respectable. S'il brise des idoles, ce n'est pas pour en faire naître de nouvelles mais pour détruire cette idolâtrie aberrante qui imprègne la conscience générale. Ce qui le distingue radicalement des surréalistes, c'est qu'il ne croit pas à un niveau merveilleux et à son hypothétique libération. Car pour lui, ce n'est pas l'homme qui est à l'image de la société mais bien la société qui est à l'image de l'homme ${ }^{10}$.

En continuum, les textes des chansons de Zappa dressent un bilan social désastreux. L'humanité semble irrécupérable; Zappa semble fataliste. II ne faut pas y déceler un caractère misanthrope; au contraire, Zappa apparaît plutôt philanthrope dans son acharnement à fustiger les déviations de ses contemporains. II faut l'avoir vu, soir après soir, lors de ses interminables séries de concerts, inviter les spectateurs à participer sur scène à de délirants rituels de défoulement transgressif qu'il dirige avec maestria. Aucune censure : notre homme s'est toujours amusé avec la fascination absolve qu'exercent les interdits.

Michel Onfray à propos d'une esthétique cynique : «[...] La parole ludique ef joyeuse, le happening pédagogique, l'usage du monde comme d'une scène et autres manières d'écrire puis de philosopher avec le corps qui définissent autant de péchés mortels dans la corporation [... $]^{11}$ ". Zappa s'y trouve.

L'humour de ce compositeur fut inégalé. Satie, peut-être.

Advance Romance : Zappa, intemporel, a toujours eu un peu d'avance sur le retard des autres...
10. One Side Fits All, Rémi Raemackers, Monaco, Éditions la Mémoire et la Mer, 2003, p. 166.

11. Archéologie du présent, Manifeste pour une esthétique cynique, op. cit., p. 16. 


\section{Particularités maladives}

Zappa fut aussi un homme d'affaires remarquable. Vous connaissez beaucoup de musiciens à avoir aussi courageusement assumé l'aspect "économique" de leur œuvre? Bien sûr l'argent ennuie, mais nous n'y échappons pas. Bien sûr l'argent rabote tout, mais il y aura toujours quelques têtes dures qui appelleront au dépassement. Zappa fut de celles-là.

La notion d'industrie culturelle et l'obsession cauchemardesque de notre société pour une culture "rentable" nivelle déjà, de façon embryonnaire, le paysage artistique des Golden Sixties lorsque Zappa jaillit en provocateur. Avec urgence, il a alors sonné l'alarme avec ses alliages vitrioliques de mots blasphématoires et de musique "fission" là l'opposé du terme fusion, car Zappa ne réunit pas, il heurte, il provoque une fission). Difficile à cataloguer, impossible à épingler, échappant à tous les schémas de rentabilité mis en place par les magnats de cette industrie naissante, il fut rapidement catalogué comme le paria de service, pouilleux faire-valoir de poulains plus propres et plus rentables. Au mieux, il était celui avec qui l'argent n'était qu'à perdre dans un happening dada finissant en émeute.

Pause :

Il a suffi à l'argent de convaincre que la consommation établirait l'égalité pour que nul ne puisse plus prétendre que l'égalité s'établirait contre la consommation que permet l'argent. Entre toutes les victoires qu'on pouvait craindre de voir l'argent remporter, celle-ci est sans doute la plus lourde de conséquences. Remportant cette victoire, l'argent a permis que l'emporte avec lui toute politique qui se réclamait de lui. Remportant cette victoire ensemble, c'est dès lors l'argent et la politique que nul ne sait plus comment distinguer' ${ }^{12}$.

Zappa n'est pas dupe : il joue dru dans les plates-bandes des rois du showbizz et revendique un droit absolu à la liberté de parole tout en commentant ouvertement le libertinage incestueux auquel se livrent les pouvoirs politiques, financiers et religieux. Résultat : ses relations avec les majors de l'industrie du disque et la RIAA (Recording Industry Association of America) ont toujours été houleuses... Zappa, là aussi, a dû combattre à la manière d'un don Quichotte farfelu ces sinistres géants qui n'en finissaient plus d'enterrer l'invention au profit du gain. Malgré tout, pendant une dizaine d'années, la créativité des Mothers of Inventions fut à son comble. De 1965 à 1975, la production sur disque de Zappa est spectaculaire (vingt disques dans cette seule décennie). Dès 1969, en réaction au crétinisme ambiant, il fonde sa propre compagnie de disque, Bizarre Productions /un label semi-indépendant du major MGM; Bizarre est le premier label que Zappa a créé ; d'autres suivront, permettant successivement une totale indépendance). Voici le petit manifeste qui accompagne alors les productions discographiques de ce jeune label: "We make records that are a little different. We present musical and sociological
12. SURYA, Michel (2000), De l'argent, La ruine du politique, Paris, Éditions Payot \& Rivages, p. 14. 
material which the important record companies would probably not allow you to hear. Just what the world needs... another record company. ${ }^{13}$

L'obsession monomaniaque du profit à tout prix est une particularité maladive de notre société marchande; elle est profondément incompatible avec les impératifs de la création. Difficile d'être un artiste et de vendre sa musique... Un défi que Zappa releva avec une vigueur prodigieuse.

Au moment où l'industrie mondiale du disque affronte la plus sérieuse crise de sa pénible histoire, celui qui s'est battu sans relâche contre cette burlesque entreprise d'arnaque semble désormais faire figure de visionnaire. Toutes ces années d'affrontements ininterrompus mis en œuvre par Zappa n'auront pas été inutiles : de petites maisons "différentes" naissent à tous les jours, créant ainsi une énorme pression sur les conglomérats de l'industrie (Ambiances Magnétiques, à Montréal, est l'une de celles-là et existe depuis maintenant vingt ans).

Souhaitons-nous, à la mémoire de Zappa et pour notre bon plaisir, l'implosion de cet ordre commercial nihiliste.
13. Traduction : «Nous faisons des disques quelque peu différents. Nous proposons un contenu musical et sociologique que les compagnies de disques importantes ne vous permettraient pas d'entendre. Ce que le monde attendait... Une autre compagnie de disque..."

\section{Clore la modernité...}

Si le syncrétisme brillant de John Zorn est spécifiquement associé à la postmodernité, l'éclectisme chercheur de Zappa a davantage ses racines dans la modernité. Mais il est peut-être le compositeur qui a clos la modernité musicale en s'ouvrant si largement aux possibilités inouïes de la rupture stylistique :

Là où d'autres musiciens de génie ont fait exploser le système musical, donnant souvent naissance à des myriades de météorites, Zappa, lui, l'a fait imploser, l'a porté à son point d'incandescence maximum, l'a miné de l'intérieur, a "viralisé" les codes musicaux, a semé ses gènes perturbateurs dans la chaîne d'un $A D N$ sonore vers quoi tend la codification. Il est celui par qui la musique du XXe siècle s'affranchit définitivement des normes pour s'ouvrir à un espace sans limite. II incarne l'ultime résistance à la redondance et à la tautologie formelles. II aura, en fin de compte, rendu à la musique son occulte pouvoir libérateur, lors même qu'elle n'était plus qu'un système de signes, le rejeton du langage ${ }^{14}$.

14. One Side Fits All, op. cit., p. 17.

En musicien courageux qu'il était, Zappa a fait de son corpus musical une constante et volontaire transgression des styles : rock, improvisation, musique concrète, rhythm \& blues, musique de chambre, doo-wop, free jazz, etc. Pionnier de la transgression et du téléscopage stylistique, il est un des seuls à avoir franchi autant de frontières, le premier à avoir permis et soutenu avec brio une musique franchement hétérogène. Un décloisonnement nécessaire qui renvoie les puristes sectaires aux dernières loges du néolithique musical, là où il y a encore une foule désolante.

Zappa méprisait les puristes; il était un musicien talentueusement éclectique. II ne s'agit pas ici d'un éclectisme de dérive, n'oubliez pas que les musiques de Zappa 
ont toujours été profondément les siennes : rigoureuses, parfaitement originales, immédiatement identifiables. Cette volonté transgressive (et l'unicité de son œuvre) doit être perçue "avant tout dans le refus forcené de toute inféodation ou allégeance aux règles de tel ou tel style, genre, école ou mode, et l'insistance à développer sa propre démarche "libertaire" contre toute facilité, qu'elle soit commerciale, stratégique, militante ou spectaculaire ${ }^{15}$.

15. Ibid., p. 13.

Zappa était un homme irréductible et libre.

\section{Joie du rétroviseur}

Ce texte arrive à une époque où j'ai passablement laissé mon intérêt pour Zappa s'émousser. Je ne le croise plus, ou rarement. Les quelques furtives retrouvailles ont presque toujours lieu avec le corpus de la première décennie, là où les Mothers of Invention déjantaient à chaque détour, entre 1965 et 1975. J'entretiens ce sentiment : depuis sa disparition, Zappa Isa notoriété, sa popularité et l'intérêt que suscitait l'ensemble de son étonnante production) sombre lentement mais sûrement dans cette phase de latence semblable à l'état d'engourdissement qu'induit la digestion, processus enzymatique impliquant une perte radicale d'appétit à la suite d'un banquet réussi (le corpus de l'ensemble des travaux de Zappa n'estil pas un festin d'une remarquable luxuriance?).

L'intense déflagration produite par l'imaginaire passionné de cet artiste engagé semble plus que jamais à contre-courant du laisser-aller pseudo militant des milieux artistiques actuels, sages et apolitiques. Plusieurs des jeunes musiciens que je croise, où avec qui je travaille, ne connaissent absolument pas Zappa : "Ah ouais, l'Américain qui créait un mélange de prog et de jazz-rock un peu weird... », etc. Dommage.

Ce début de millénaire est trop sage. Combien de faux fomentateurs pétrifiés pour quelques miettes d'éternité?

Rétrospectivement, Zappa nous indique qu'un créateur a toutes les libertés, qu'il a tous les privilèges, et qu'il a le devoir de troubler l'ordre social, par nature statique et conservateur. Aux jeunes musiciens et compositeurs, Zappa dit encore et toujours ceci : il faudrait vous rebeller davantage, cesser d'avoir peur, avoir d'autres préoccupations que la seule réussite. Mieux encore : essayer de dire ce qui n'est pas dit, ou dire autrement ce qui n'a pas été dit d'une manière convaincante. Et surtout, ne jamais dissocier art et politique.

"Register to vote!", écrivait-il (ordonnait-il!) sur les pochettes de ses disques; proposition à l'usage des jeunes mélomanes dépolitisés; geste lucide d'un artiste sensible au désastre politique de son pays. Ce message n'est-il pas toujours d'une urgente actualité ? 


\section{Geste indigeste}

Avec tous, Zappa était intransigeant, de même qu'avec lui-même, envers et contre tous. À mon tour.

II serait triste de rater cette occasion.

Comprenez ceci : Zappa est avant tout un guitariste mortellement séduit par le rhythm \& blues, un rockeur mélomane, un virtuose de la scène et du studio. Quand à son répertoire néoclassique (par exemple, l'ensemble du corpus interprétés par le London Symphony Orchestral, disons simplement que l'esthétique de Zappa n'était pas adapté à ce type de sonorités avachies. Les seules œuvres orchestrales dignement représentatives de son esthétique se retrouvent sur les disques "Studio Tan", "Grand Wazoo» et "Orchestral Favorites". Zappa sans batterie, ça ne fonctionne tout simplement pas (sauf quelques exceptions, "The Yellow Shark", notamment).

La quintessence de son travail, son véritable génie, se situe du côté de ce qu'il a écrit pour ses propres ensembles. Ensembles avec lesquels le guitariste nous a démontré quel prodigieux rythmicien il fut. Si vous voulez écouter Zappa, écoutez les Mothers of Invention (toutes époques confondues) et laissez de côté l'Ensemble Intercontemporain. Vous pourrez toujours y revenir si vous jugez encore l'exercice utile. Zappa n'avait nul besoin de s'exhiber en compagnie de Boulez. Soyons indulgents pour cette association contre-nature. Voyons-y le résultat d'une obsession quasi irrationnelle : le désir d'une reconnaissance "officielle"; la livraison d'une preuve, s'il en faut, que notre homme était un "vrai» compositeur... Étonnante erreur stratégique pour cet habile tacticien : plutôt qu'une porte ouverte en direction du cénacle des compositeurs désignés immortels, Zappa s'est égaré dans une esthétique néoclassique qui n'apportait rien de nouveau à un catalogue par ailleurs très innovateur. En cédant à ce désir discutable, Zappa mettra un terme définitif à ce qui fut, sans conteste, sa période la plus prolifique.

\section{Freak Out et nostalgie}

It isn't necessery to imagine the world ending in fire or ice - there are two other possibilities: one is paperwork, and the other is nostalgia. When you compute the length of time between The Event and The Nostalgia for the Event, the span seems to be about a year less in each cycle. Eventually within the next quarter of a century, the nostalgia cycles will be so close together that people will not be able to take a step without being nostalgic for the one they just took. At that point, everything stops. Death by Nostalgia. ${ }^{10}$
16. Traduction : «Nul besoin d'imaginer une fin du monde par le feu ou la glace il y a deux autres possibilités : la paperasserie et la nostalgie. Si vous mesurez le temps qui s'écoule entre l'Événement et la Nostalgie de cet Événement, vous constatez une réduction du délai de l'ordre d'un an à 
En 1965, Cage avait 53 ans, Miles 39, et Zappa 25. Au moment précis où les États-Unis s'engageaint ouvertement dans le désastre du Viêtnam, trois générations successives de musiciens aux esthétiques divergentes se côtoyaient dans un tumulte esthétique. Aucune collaboration n'a été possible, mais un entrelacs extrêmement significatif s'offrait alors aux mélomanes d'Amérique : Cage ne cessa d'inventer, Rozart Mix, Electronic Music for Piano et Variations V; Miles amorçait la prodigieuse période de son Quintet, permettant entre autres l'éclosion d'une section rythmique inouie (Tony Williams innovait à chaque détour); de son côté, Zappa enregistra le premier disque des Mothers of Invention, étonnant disque double qui offrait un avant-goût stupéfiant de ce que sera sa production future. Vingthuit années plus tard, le plus jeune des trois s'éteignait prématurément.

Dommage, il aurait fait un excellent président. Freak Out! chaque nouveau cycle. Éventuellement, dans le prochain quart de siècle, les cycles de la nostalgie seront si rapprochés que les gens ne pourront plus faire un pas sans ressentir la nostalgie du précédent. À ce momentlà, tout s'arrête. C'est la mort par nostalgie. » 


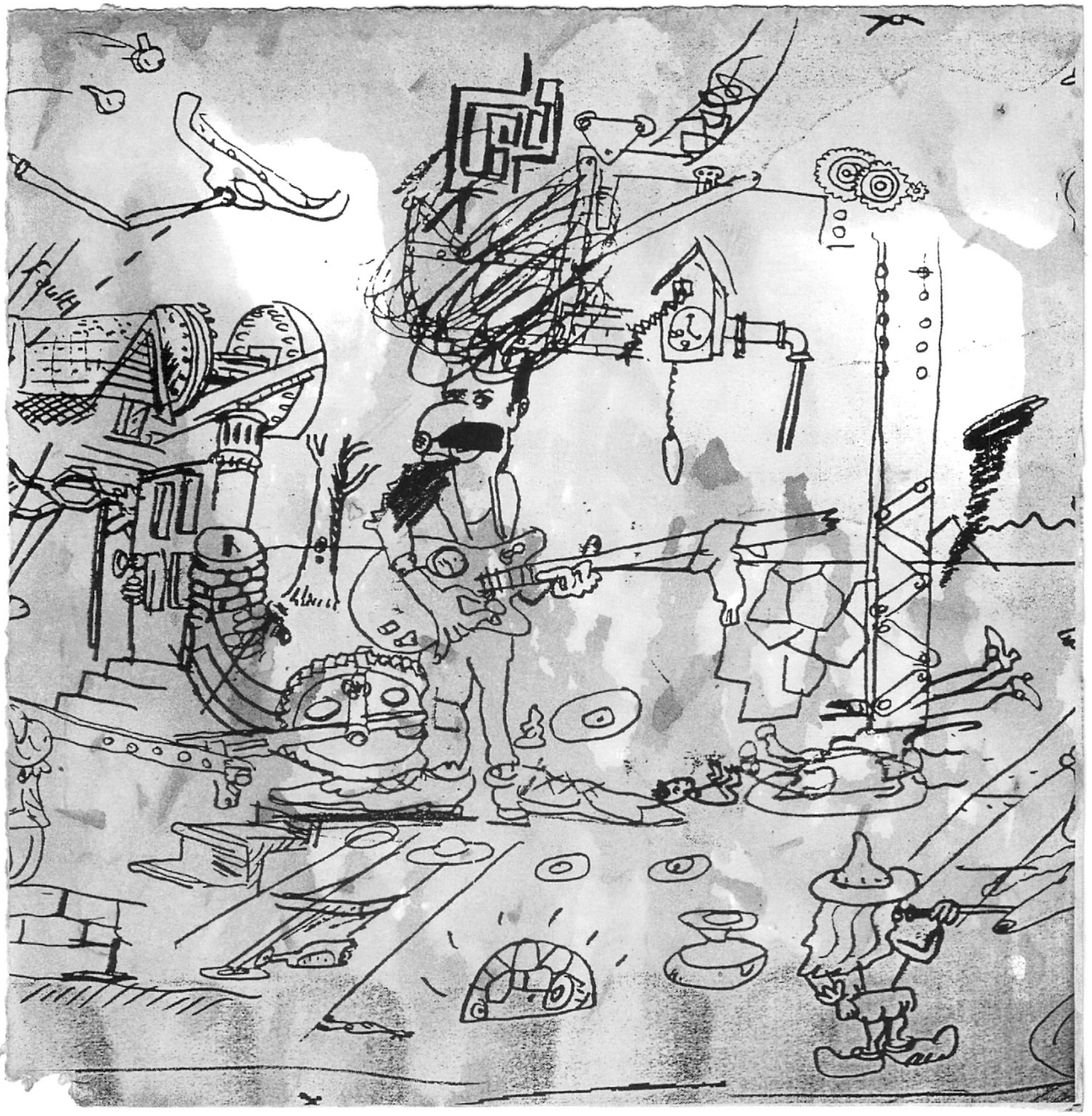

